\title{
ON PROPERLY EMBEDDING PLANES IN ARBITRARY 3-MANIFOLDS
}

\author{
E. M. BROWN AND C. D. FEUSTEL
}

\begin{abstract}
We prove an analog of the loop theorem for an arbitrary noncompact 3-manifold. In particular, we show that the existence of a "nontrivial" proper map of a plane into a 3-manifold implies the existence of a nearby nontrivial embedding of a plane into the 3-manifold.
\end{abstract}

Introduction. In [2] we showed that if $M$ is an eventually end-irreducible 3-manifold and $f: \mathbf{R}^{2} \rightarrow M$ is a proper essential map, then there is a proper essential embedding $g: \mathbf{R}^{2} \rightarrow M$. In this paper we remove the restriction to manifolds which are eventually end-irreducible. This permits us to choose the image of $g$ to lie in a preassigned neighborhood of the image of $f$. This could not be done with the weaker theorem, for even when $M$ is eventually end-irreducible, a regular neighborhood of $f\left(\mathbf{R}^{2}\right)$ need not be. We also extend the theorem in another direction; roughly, we show that if a normal subgroup of the fundamental group of the complement of a compact set in $M$ is given and large loops around the origin in $\mathbf{R}^{2}$ are mapped by $f$ to homotopy classes not in the subgroup, then $g$ can be chosen to have the same property.

The proof of the main theorem, (2.2), parallels that of [2]. At one point, [2] makes major use of the eventually end-irreducible hypothesis. In this paper we prove Lemma (2.1) to substitute where eventually end-irreducible was used before. We believe the technique used in Lemma (2.1) is new. We expect it to be generally applicable to 3-manifolds which are not eventually end-irreducible.

1. Notational conventions and a preliminary lemma. We work in the category of simplicial complexes and piecewise linear maps. A map $f: X \rightarrow Y$ is proper if $f^{-1}(C)$ is compact for every compact $C \subset Y$. If $X \subset Y$ we use $\operatorname{Fr}(X)$ to mean the frontier of $X$ in $Y$ and $\mathrm{Cl}(X)$ to mean the closure of $X$ in $Y$. If $M$ is a manifold, we use $\partial M$ for the boundary of $M$. We follow Waldhausen's convention [6] on regular neighborhoods; specifically, if $X \subset Y$, choose a triangulation of $Y$ in which all previously mentioned subspaces are subcomplexes, and let $U(X)$ be the simplicial neighborhood of $X$ in the second barycentric subdivision.

If $M$ is a 3-manifold, an exhausting sequence for $M$ will be a sequence $\left\{M_{n}\right\}$ of compact 3-manifolds in $M$ with $M_{n} \subset M_{n+1}-\operatorname{Fr}\left(M_{n+1}\right), M_{n} \cap \partial M$ a 2-manifold,

Received by the editors November 24, 1982 and, in revised form, June 6, 1984.

1980 Mathematics Subject Classification. Primary 57M35.

Key words and phrases. Proper map, proper embedding, loop theorem, plane. 
and $\cup_{n} M_{n}=M$. The word "proper" is used in two senses; here, where applicable, we mean both. In particular, a 2-manifold $F$ is properly embedded in a 3-manifold $M$ if the inclusion map $F \rightarrow M$ is proper and $F \cap \partial M=\partial F$. If $F$ is a 2-manifold properly embedded in a 3-manifold $M$, Jaco denotes the result of cutting $M$ over $F$, i.e. $\mathrm{Cl}(M-U(F)), \alpha_{F}(M)$. We use $\alpha(F, M)$ for this to avoid double subscripts. If $M, N(M \subset N)$ are 3-manifolds and $F \subset N$ is a 2-manifold with $F \cap \operatorname{Fr}(M)=\partial F$, we also write $\alpha(F, M)$ for $M \cup U(F)$ (we still refer to this as cutting $M$ over $F$ ). We also write $\alpha(F, M)$, where $F$ is a finite family of 2-manifolds and we are cutting over them in sequence. When the order in which we cut matters, we give $F$ as an ordered family.

We shall write our fundamental groups without basepoint, as our use of fundamental groups will be independent of the choice of basepoint. If $f: A \rightarrow M$ is a map of a 1-sphere into $M$, we use [ $f$ ] for the homotopy class in $\pi_{1}(M)$ of $f$ with some choice of basepoint and some orientation of $S^{1}$. Notice that if $G$ is a normal subgroup of $\pi_{1}(M)$ then $[f] \notin G$ makes sense. An unlabeled map between fundamental groups will always be induced by inclusion.

We use the loop theorem [5] without further reference.

LeMma (1.1) Let $M$ be a 3-manifold and let $M_{0}$ be a compact submanifold. There is an exhausting sequence $\left\{M_{m}\right\}$ for $M$, with $M_{0} \subset M_{m}$, and for each $m$ there is a finite family $\mathscr{D}_{m}$ of disks properly embedded in $M_{m}-M_{0}-\partial M$ so that $\operatorname{Fr}\left(\alpha\left(\mathscr{D}_{m}, M_{m}\right)\right)$ is incompressible in $M-M_{0}$.

We do not assert that $\left\{\alpha\left(\mathscr{D}_{m}, M_{m}\right)\right\}$ exhausts $M$; that is, generally, impossible. The point is that all the members of $\mathscr{D}_{m}$ are contained in $M_{m}$.

Proof. Let $\left\{M_{m}^{\prime}\right\}$ be an exhausting sequence for $M$. Suppose, by induction, that for all $1 \leqslant m<n$ we have chosen $M_{m}$ and $\mathscr{D}_{m}$ as above and, in addition, $M_{m}^{\prime} \subset M_{m}$ (this last is so that the sequence we end up with exhausts $M$ ). If $M_{n}$ is any compact manifold with $M_{n-1} \cup M_{n}^{\prime} \subset M_{n}-\operatorname{Fr}\left(M_{n}\right)$, it is a standard argument that one can construct a finite sequence of disks $\mathscr{D}_{n}=\left(D_{1}, \ldots, D_{k}\right)$ in $M-M_{0}$ so that $\operatorname{Fr}\left(\alpha\left(\mathscr{D}_{n}, M_{n}\right)\right)$ is incompressible in $M-M_{0}$. We may not, however, be able to choose all the disks in $M_{n}$. We show that if $M_{n}$ is chosen appropriately, this can be done; choose $M_{n}$ and $\mathscr{D}_{n}$ so that $\mathscr{D}_{n}$ has the least number of disks possible not contained in $M_{n}$. Let $D_{i}$ be the first in the sequence so that $D_{i}$ is not contained in $M_{n}$. We may assume that $D_{1}, \ldots, D_{i-1}$ are disjoint and for $j<i$ that $D_{i}$ meets $U\left(D_{j}\right)$ in disks parallel to $D_{j}$, i.e. in disks whose boundaries are essential in the annulus $U\left(D_{j}\right) \cap \operatorname{Fr}\left(M_{n}\right)$. Let $M_{n}^{\prime \prime}=M_{n} \cup U\left(D_{i}\right)$. Then $\alpha\left(\left(D_{1}, \ldots, D_{i}\right), M_{n}\right)$ can be constructed from $M_{n}^{\prime \prime}$ by cuts parallel to the disks $D_{1}, \ldots, D_{i-1}$. It follows that all of $D_{1}, \ldots, D_{k}$ are contained in $M_{n}$ and the lemma is proved.

If $D$ is a properly embedded disk in a 3-manifold $M$ there is a homeomorphism of $U(D)$ onto $D \times[-1,1]$ carrying $U(D) \cap \partial M$ onto $\partial D \times[-1,1]$. We identify via such a homeomorphism and use $U(D)$ and $D \times[-1,1]$ interchangeably. In Lemma (1.1) we may assume the disks chosen so that if $D \in \mathscr{D}_{m}$ and $E \in \mathscr{D}_{n}, m<n$, then $U(E) \cap M_{m} \subset U\left(\cup \mathscr{D}_{m}\right)$ and $U(E) \cap U(D)=D \times F$, where $F \subset(-1,1)$ is a finite set of closed intervals. We may further assume that if $p \in E \cap U(D)$, then the 
product line $\{p\} \times[-1,1]$ of $U(E)$ is contained in the product line $\{p\} \times[-1,1]$ of $U(D)$. We make these assumptions when we use Lemma (1.1).

2. The main lemma and the theorem. If $C$ is a compact subset of $M$, by a complementary domain of $C$ we mean the closure of a component of $M-C$.

LeMma (2.1). Let $M$ be a 3-manifold and let $f: \mathbf{R}^{2} \rightarrow M$ be a proper map. Let $C \subset M$ be compact, let $A$ be a complementary domain of $C$, and let $G$ be a normal subgroup of $\pi_{1}(A)$. Suppose $D_{0} \subset \mathbf{R}^{2}$ is a disk, $f\left(\mathbf{R}^{2}-D_{0}\right) \subset A$, and $\left[f \mid \partial D_{0}\right] \notin G$. Then there exists an exhausting sequence $\left\{M_{m}^{\prime}\right\}$ for $M$, an exhausting sequence $\left\{D_{m}\right\}$ of disks in $\mathbf{R}^{2}$, and a proper map $g: \mathbf{R}^{2} \rightarrow M$ so that $g\left(\mathbf{R}^{2}-D_{0}\right) \subset A,\left[g \mid \partial D_{0}\right] \notin G$, and $D_{m}$ is a component of $g^{-1}\left(M_{m}^{\prime}\right)$.

PROOF. We may assume that $f\left(\mathbf{R}^{2}\right) \cap \partial M$ is empty. It is sufficient to prove that if $L \subset M$ is any compact submanifold containing $C$ then there exists a proper map $g: \mathbf{R}^{2} \rightarrow M$ and a compact submanifold $N$ of $M$ containing $L$ so that $f^{-1}(L)=$ $g^{-1}(L), f\left|f^{-1}(L)=g\right| f^{-1}(L), g^{-1}(L)$ is contained in a component of $g^{-1}(N)$, and that component is a disk.

Suppose $L$ is given; choose a compact submanifold $M_{0}$ of $M$ so that:

(1) $f^{-1}(L)$ is contained in a single component of $f^{-1}\left(M_{0}\right)$.

Subject to the restrictions on $g$ above, assume $f$ has been altered so that $f^{-1}\left(M_{0}\right)$ has the least number of components possible. Now choose $\left\{M_{m}\right\}$ and $\left\{\mathscr{D}_{m}\right\}$ as in Lemma (1.1). Altering $f$ outside $f^{-1}\left(M_{0}\right)$ by a proper homotopy and choosing a subsequence of $\left\{M_{m}\right\}$, we may further assume:

(2) $f$ is in general position with respect to $M_{m}$ and $\mathscr{D}_{m}$ for all $m$.

(3) $f\left(\mathbf{R}^{2}\right) \cap(D \times[-1,1])=\left(f\left(\mathbf{R}^{2}\right) \cap D\right) \times[-1,1]$ for all $D \in \mathscr{D}_{m}$.

(4) $f^{-1}\left(M_{m}\right)$ is contained in a disk in $f^{-1}\left(M_{m+1}\right)$ for $m=0,1, \ldots$

Let $N_{m}=\alpha\left(\mathscr{D}_{m}, M_{m}\right)$. Let $A_{0}=B_{0}$ denote the component of $f^{-1}\left(M_{0}\right)$ which contains $f^{-1}(L)$. Let $A_{m}$ (respectively, $B_{m}$ ) denote the component of $f^{-1}\left(M_{m}\right)$ (respectively, $f^{-1}\left(N_{m}\right)$ ) which contains $A_{m-1}$ (respectively, $B_{m-1}$ ). It follows from (2) and (3) that $A_{m}$ and $B_{m}$ are disks-with-holes, and from (4) that $f^{-1}\left(M_{m-1}\right) \subset A_{m}$, for $m=1,2, \ldots$. A component of $\partial A_{m}$ (respectively, $\partial B_{m}$ ) is called an inner boundary component if the disk it bounds in $\mathbf{R}^{2}$ does not contain $A_{m}$ (respectively, $B_{m}$ ).

We show that $f$ can be altered so that all inner boundary components of some $B_{n}$ disappear. Then $B_{n}$ is a disk and contains $f^{-1}(L)$, so $N=N_{n}$ will prove the lemma. Let $\lambda$ be an inner boundary component of $B_{m}$. If $f(\lambda)$ is an inessential loop in $\operatorname{Fr}\left(N_{m}\right)$, then we can alter $f$ to carry a small neighborhood of the disk bounded by $\lambda$ into a collar neighborhood of $\operatorname{Fr}\left(N_{m}\right)$ (which eliminates $\lambda$ from the list of inner boundary components of $B_{m}$ ). This will certainly be the case if the disk bounded by $\lambda$ contains no component of $f^{-1}\left(M_{0}\right)$. We show that this last is true for $m$ sufficiently large; indeed, choose $n$ so that $B_{n}$ contains as many components of $f^{-1}\left(M_{0}\right)$ as possible.

By (2) and (3), the inner boundary components of $B_{n}$ are of three kinds:

(5) components which are also inner boundary components of $A_{n}$,

(6) components which miss $\partial A_{n}$, 
(7) components made up of arcs which $f$ maps properly into $U \mathscr{D}_{n} \times\{-1,1\}$ alternating with arcs of $\partial A_{n}$.

Let $\lambda$ be an inner boundary component of $B_{n}$. We show that the disk $E$ bounded by $\lambda$ contains no points of $f^{-1}\left(M_{0}\right)$. Since $f^{-1}\left(M_{0}\right)$ is contained in $A_{n}$, this is obvious if $\lambda$ is of type (5). If $\lambda$ is of type (6) then $f(\lambda) \subset D \times\{-1,1\}$ for some $D \in \mathscr{D}_{n}$ : since $f^{-1}\left(M_{0}\right)$ has the least number of components possible, it again follows that $E$ misses $f^{-1}\left(M_{0}\right)$. Suppose $\lambda$ is of type (7) and $E$ contains a component $K$ of $f^{-1}\left(M_{0}\right)$. By our choice of $n, K \not \subset B_{n+1}$, and since $B_{n} \subset B_{n+1}$, there is an inner boundary component $\mu$ of $B_{n+1}$ with $K$ contained in the disk bounded by $\mu$. Again, since $B_{n} \subset B_{n+1}, \mu \subset E$. By (4), $A_{n}$ is contained in a disk in $A_{n+1}$; since $f(\mu) \subset f(E) \subset$ $\operatorname{int}\left(M_{n+1}\right), \mu$ is of type (6). We have seen that no such curve of type (6) exists, and the lemma is proved.

If $f: \mathbf{R}^{2} \rightarrow M$ is a proper map, then for any compact $C \subset M$ there is a complementary domain $A$ of $C$ and a disk $C \subset \mathbf{R}^{2}$ so that $f\left(\mathbf{R}^{2}-D\right) \subset A$. If, for some choice of $C,[f \mid \partial D]$ is not trivial in $\pi_{1}(A)$, then $f$ is an essential map. The following theorem will show, in particular, that a proper essential map may be replaced by a proper essential embedding.

THEOREM (2.2). Let $M$ be a 3-manifold and let $f: \mathbf{R}^{2} \rightarrow M$ be a proper map. Suppose, for some compact $C \subset M$, that if $D \subset \mathbf{R}^{2}$ is the disk and $A$ the complementary domain of $C$ with $f\left(\mathbf{R}^{2}-D\right) \subset A$, then $[f \mid \partial D] \notin G$ for $G$ a normal subgroup of $\pi_{1}(A)$. Then for any neighborhood $U$ of $f\left(\mathbf{R}^{2}\right)$ there is a proper embedding $g: \mathbf{R}^{2} \rightarrow M$ and a disk $E \subset \mathbf{R}^{2}$ so that $g\left(\mathbf{R}^{2}\right) \subset U, g\left(\mathbf{R}^{2}-E\right) \subset A$, and $[g \mid \partial E] \notin G$.

Proof. Choose a triangulation of $M$ so that $U\left(f\left(\mathbf{R}^{2}\right)\right) \subset U$. We may replace $M$ by $U\left(f\left(\mathbf{R}^{2}\right)\right), C$ by $C \cap U\left(f\left(\mathbf{R}^{2}\right)\right), A$ by the complementary domain $A^{\prime}$ of $C \cap U\left(f\left(\mathbf{R}^{2}\right)\right)$ in $U\left(f\left(\mathbf{R}^{2}\right)\right)$ which contains $f\left(\mathbf{R}^{2}-D\right)$, and $G$ by $i_{*}^{-1}(G)$, where $i: A^{\prime} \rightarrow A$ is the inclusion map. Thus, it is sufficient to prove the theorem without the condition $g\left(\mathbf{R}^{2}\right) \subset U$.

Returning to the original notation we use Lemma (2.1) to find an exhausting sequence $\left\{M_{i}\right\}$ for $M$ and a nested sequence of disks $\left\{D_{i}\right\}$ in $\mathbf{R}^{2}$ so that $C \subset M_{1}$, $D \subset D_{1}$, and $D_{i}$ is a component of $f^{-1}\left(M_{i}\right)$. We assume that $f$ is in general position with respect to $\partial M_{i}$ for every $i$. Since $D \subset D_{i}$, it follows that $f\left(\partial D_{i}\right) \subset A$ for every $i$. As in [2] we may use Stallings' proof of the loop theorem [5] to choose a sequence of embedded disks $\left\{E_{k}\right\}$ in $M-\partial M$, and a sequence of simple loops $\left\{\lambda_{j}\right\}$ in $A$ so that:

(1) $\cup_{k \geqslant i} \pi_{0}\left(\operatorname{Fr}\left(M_{i}\right) \cap E_{k}\right)$ is a finite set $\left(\pi_{0}(X)\right.$ is the set of path components of $X)$.

(2) $\left[\lambda_{j}\right] \notin G \subset \pi_{1}(A)$.

(3) If $k \geqslant j$ then $\lambda_{j}$ is a component of $\operatorname{Fr}\left(M_{j}\right) \cap E_{k}$, and the disk in $E_{k}$ bounded by $\lambda_{j}$ contains $\lambda_{j-1}$.

For each $j<k$ we let $A(j, k)$ be the annulus in $E_{k}$ bounded by $\lambda_{j}$ and $\lambda_{j+1}$. Then for $i<j$ we set $J(i, j)=\left\{k \mid M_{i} \cap A(j, k)=\varnothing\right\}$.

LEMMA. For each $i$ there exist arbitrarily large integers $m$ so that for all $r$, $\bigcap_{j=m}^{m+r} J(i, j) \neq \varnothing$. (Intuitively for fixed $i$ annuli far enough out on $E_{k}$ miss $M_{i}$.) 
Proof. Let $m(i)$ be one greater than the number of elements in the finite set $\bigcup_{k \geqslant i} \pi_{0}\left(\operatorname{Fr}\left(M_{i}\right) \cap E_{k}\right)$. We first show that for fixed $i$ any $m(i)$ of the sets $J(i, j)$ contain all but a finite number of the integers. Indeed, if $k \notin J(i, j)$, then $\operatorname{Fr}\left(M_{i}\right) \cap$ $A(j, k) \neq \varnothing$. This cannot happen for $m(i)$ distinct values of $j$ since $E_{k} \cap \operatorname{Fr}\left(M_{i}\right)$ has fewer than $m(i)$ components.

Suppose for some $i$ the lemma is false. Then for all sufficiently large $m$, there exists $r$ with $\bigcap_{j=m}^{m+r} J(i, j)=\varnothing$. Let $j(1)>i$ be sufficiently large and choose $r(1)$ so that $\bigcap_{j=j(1)}^{j(1)+r(1)} J(i, j)=\varnothing$. Inductively choose $j(s), r(s), s=2, \ldots, m(i)$, so that $j(s)>j(s-1)+r(s-1)$, and $\bigcap_{j=j(s)}^{j(s)+r(s)} J(i, j)=\varnothing$. Let $k>j(m(i))+r(m(i))+$ 1. Then for each $s=1,2, \ldots, m(i)$, there exists $j^{\prime}(s), j(s)<j^{\prime}(s) \leqslant j(s)+r(s)$, so that $k \notin J\left(i, j^{\prime}(s)\right)$. Then $k \notin \bigcup_{s=1}^{m(i)} J\left(i, j^{\prime}(s)\right)$, which contradicts the assertion of the last paragraph. The lemma is proved.

Notice that if $k \in \bigcap_{j=m}^{m+r} J(i, j)$, then the annulus on $E_{k}$ bounded by $\lambda_{m}$ and $\lambda_{m+r}$ does not meet $M_{i}$.

We will construct a singular plane in $M$ whose only singularities are double curves. We realize this plane as the union of an embedded disk $A_{0}$ and embedded annuli $A_{i}$ so that $A_{i} \cap A_{j} \neq \varnothing$ iff $|i-j|<2$. Moreover, $A_{i} \cap \partial A_{i+1}=\partial A_{i} \cap A_{i+1}$ is a single component of the boundary of each.

Let $A_{0}$ be the disk bounded by $\lambda_{1}$ on $E_{2}$. Let $i(1)$ be an integer such that $A_{0} \subset M_{i(1)}$. Let $m(1)$ be an integer such that, for all $r, \bigcap_{j=m(1)}^{m(1)+r} J(i(1), j)$ is not empty. The existence of $m(1)$ follows from our lemma.

Let $A_{1}$ be the annulus bounded by $\lambda_{1}$ and $\lambda_{m(1)}$ on $E_{m(1)+1}$. Let $i(2)$ be an integer such that $A_{1} \subset M_{i(2)}$, and let $m(2)>m(1)$ be an integer such that for all $r$, $\bigcap_{j=m(2)}^{m(2)+r} J(i(2), j) \neq \varnothing$. Choose $k(2) \in \bigcap_{j=m(1)}^{m(2)} J(i(1), j)$, which is not empty by our choice of $m(1)$. Then let $A_{2}$ be the annulus on $E_{k(2)}$ bounded by $\lambda_{m(1)}$ and $\lambda_{m(2)}$. Observe that $A_{2} \cap A_{0} \subset A_{2} \cap M_{i(1)}=\varnothing$.

Now we will inductively choose $i(s), m(s), k(s)$, and $A_{s}$. First we choose $i(s)$ so that $A_{s-1} \subset M_{i(s)}$. Then we choose $m(s)>m(s-1)$ so that, for all $r$, $\bigcap_{j=m(s)}^{m(s)+r} J(i(s), j) \neq \varnothing$, which is possible by our lemma. Next $k(s)$ is chosen in $\bigcap_{j=m(s-1)}^{m(s)} J(i(s), j)$, which is not empty by our choice of $m(s-1)$. Finally, $A_{s}$ is the annulus on $E_{k(s)}$ bounded by $\lambda_{m(s-1)}$ and $\lambda_{m(s)}$. Notice that $A_{s-2} \cap A_{s} \subset M_{i(s-1)} \cap$ $A_{s}=\varnothing$ by choice of $k(s)$.

We observe that a map $f^{\prime}: \mathbf{R}^{2} \rightarrow M$ which, in polar coordinates sends $\{(r, \theta) \mid n \leqslant$ $r \leqslant n+1\}$ homeomorphically onto $A_{n}$, satisfies all the hypotheses on $f$ in the statement of the theorem. Moreover, the singularities of $f^{\prime}$ are double curves in the interior of the annuli $A_{n}$. We show how to remove these singularities by cut and paste in order to obtain the proper embedding $g$.

For $n \geqslant 1$ let $\lambda$ be a component of $\operatorname{int}\left(A_{n}\right) \cap \operatorname{int}\left(A_{n+1}\right)$. Since a boundary component of $A_{n}$ does not represent an element of $G$, it follows that $\lambda$ is essential in $A_{n}$ iff $\lambda$ is essential in $A_{n+1}$. Each essential $\lambda$ splits $A_{n}$ into two annuli: an inner one which meets $\partial A_{n-1}$, and an outer one. For each $n \geqslant 1$ in turn, choose $\lambda$ essential so that its inner annulus on $A_{n}$ contains no further essential components of $\operatorname{int}\left(A_{n}\right) \cap$ $\operatorname{int}\left(A_{n+1}\right)$. Replace $A_{n}$ by the inner annulus of $\lambda$ on $A_{n}$, and $A_{n+1}$ by the outer annulus of $\lambda$ on $A_{n+1}$. For the case $n=0$ we choose $\lambda$ innermost on $A_{0}$, essential on 
$A_{1}$. Replace $A_{1}$ by the outer annulus of $\lambda$ on $A_{1}$, and $A_{0}$ by the disk bounded by $\lambda$ on $A_{0}$. Now for every $n \geqslant 0, \operatorname{int}\left(A_{n}\right) \cap \operatorname{int}\left(A_{n+1}\right)$ consists of simple closed curves, inessential on both.

For $n=0,1,2, \ldots$ let $B_{2 n}=A_{2 n}$. Suppose $\lambda$ is an innermost simple curve on $B_{2 n}$ in $A_{2 n+1} \cap B_{2 n}$. Then $\lambda$ bounds a disk $D$ on $A_{2 n+1}$, and we may replace a slightly larger disk on $A_{2 n+1}$ in the usual way with a disk parallel to the disk bounded by $\lambda$ on $B_{2 n}$. After a finite number of such cuts we may assume that $A_{2 n+1}$ no longer meets $B_{2 n}$. It is, however, possible that $A_{2 n+1}$ now meets $A_{2 n-1}$, but only in a small neighborhood on $B_{2 n}$. In this case we replace disks on $A_{2 n-1}$ by disks parallel to disks both on $B_{2 n}$ and $A_{2 n+1}$. Thus $A_{2 n+1}$ misses both $B_{2 n}$ and $A_{2 n-1}$. Finally, using cuts, $A_{2 n-1}$ can be assumed to miss $B_{2 n}$. Now $B_{2 n-1}$ is the annulus resulting from $A_{2 n-1}$, and $\cup_{n} B_{n}$ is a nonsingular plane. If $B_{2 n+1} \cap M_{i} \neq \varnothing$, then $\left(B_{2 n} \cup B_{2 n+2}\right) \cap$ $M_{i} \neq \varnothing$. Thus, $\cup_{n} B_{n}$ is properly embedded in $M$. There is a homeomorphism of $\mathbf{R}^{2}$ onto $\cup_{n} B_{n}$ which satisfies the conditions of the theorem.

\section{REFERENCES}

1. E. M. Brown, On proper homotopy tvpe, Lecture Notes in Math., vol. 374, Springer-Verlag, Berlin and New York, 1974, pp. 41-46.

2. E. M. Brown, M. S. Brown and C. D. Feustel. On properly embedding planes in 3-manifolds, Trans. Amer. Math. Soc. 55 (1976), 461-464.

3. E. M. Brown and T. W. Tucker, On proper homotopy theory for noncompact 3-manifolds, Trans. Amer. Math. Soc. 188 (1974), 105-126.

4. D. W. Henderson, Extensions of Dehn's lemma and the loop theorem, Trans. Amer. Math. Soc. 120 (1965), 448-459.

5. J. R. Stallings, On the loop theorem, Ann. of Math (2) 72 (1960), 12-19.

6. F. Waldhausen, On irreducible 3-manifolds that are sufficiently large, Ann. of Math (2) 87 (1968), $56-88$.

Department of Mathematics, Dartmouth College, Hanover, New Hampshire 03755

Department of Mathematics, Virginia Polytechnic Institute and State University, BlacksBURG, VIRGINIA 24061 